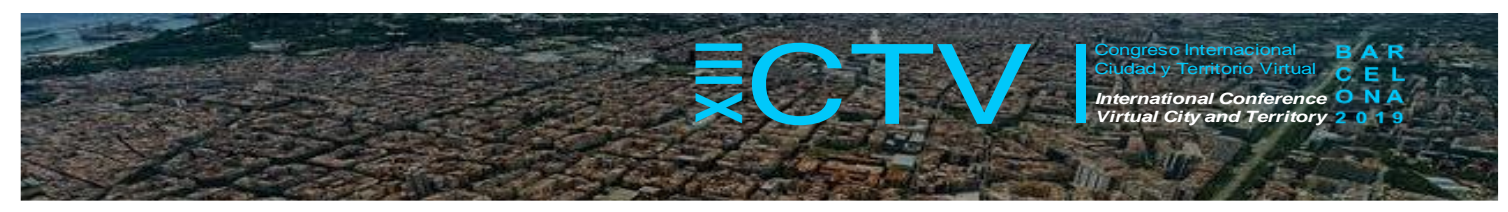

\title{
PLATAFORMA PAISAJES PEDAGÓGICOS: UN TERRITORIO VIRTUAL PARA APRENDER
}

\author{
Goñi, Ana Laura ${ }^{1 *}$; Piazza, Norma²; Payssé, Marcelo³; Inda, Hugo ${ }^{4}$ \\ Remisión inicial: 2019-05-15; Remisión definitiva: 2019-09-17; Publicación: 2019-12-21
}

Citación: Goñi, A. L. et al. (2019). Plataforma Paisajes Pedagógicos: un territorio virtual para aprender. En XIII CTV 2019 Proceedings: XIII International Conference on Virtual City and Territory: "Challenges and paradigms of the contemporary city": UPC, Barcelona, October 2-4, 2019. Barcelona: CPSV, 2019, p. 8297. E-ISSN 2604-6512. DOI http://dx.doi.org/10.5821/ctv.8297

\begin{abstract}
Resumen
El objetivo de este documento es exponer el diseño y puesta en marcha de una plataforma digital titulada Plataforma Paisajes Pedagógicos (PLAPP), disponible en http://www.plapp.edu.uy, orientada a niños en edad escolar que se construyó a partir de dispositivos tecnológicos de sensoramiento y visualización remota. La misma expone los resultados finales de una investigación sobre la percepción del paisaje local de escolares de una localidad al este de Uruguay. La fotografía digital, los vuelos de drones y las cartografías participativas se aplican como herramientas innovadoras para comprender el paisaje desde diferentes escalas y enfoques.
\end{abstract}

El equipo interdisciplinario de investigadores conformado por arquitectos, antropólogos, paisajistas y docentes, trabajó con educandos y educadores de una escuela rural como experiencia piloto. La investigación se desarrolló en un período de doce meses. Los resultados se adaptaron en un formato comunicacional y de contenido adecuado para la población escolar. Se desarrolló una plataforma digital que presenta varios puntos de observación con capacidad de visión 360 grados y con puntos sensibles que despliegan información adicional. Las estaciones cuentan con ayudas para la navegación y posibilidades de orbitar, cambiar de ubicación, ampliar y capturar la imagen, a través de menús auxiliares. Se integran fichas de vegetación nativa y de aves relacionadas al nomenclátor local, generadas por el equipo de investigadores, dibujos, textos y audios generados por los niños participantes, así como estudios de imágenes aéreas históricas del lugar, en base a fotografías aéreas del Servicio Geográfico local, de los años 1943. 1966, 1985 y una serie de fotografías satelitales más actuales, generando con la técnica del morphing un recorrido fotográfico histórico que exhibe las transformaciones del paisaje en el tiempo.

La tecnología de vehículos aéreos controlados a distancia permitió obtener video e imagen de alta calidad (4K $3840 \mathrm{x}$ 2160 pxs), desde ubicaciones normalmente inaccesibles a nivel suelo. Las tomas aéreas cubrieron áreas del territorio y generaron panoramas comprehensivos, obteniendo visiones de conjunto, referencias y nodos de control. El registro fotográfico aéreo se realizó a partir de vuelos circulares a distintas alturas y tomas panorámicas fijas con diferentes ángulos de cámara. Otra de las utilidades que se desarrolló es la generación de panoramas de varias imágenes que luego se asociaron para formar un casquete semiesférico, a los efectos de ser navegado interactivamente en la web. La imagen esférica que se despliega en pantalla resulta de la composición de 27 imágenes planas. Se configuró una versión para pantalla táctil en la que los desplazamientos son los habituales en esos dispositivos.

La estrategia metodológica está relacionada con Learning Analytics, considerando la evaluación del aprendizaje a partir del uso de nuevos dispositivos tecnológicos. Busca interpretar los datos recopilados en las actividades propuestas en el proyecto, para definir e identificar elementos problemáticos o desafíos en la apropiación y uso de los nuevos dispositivos. La aplicación del análisis de contenido y el análisis del discurso se incluye en el proyecto, capturando datos significativos de las interacciones entre los niños, el lenguaje utilizado y las nuevas tecnologías. En las actividades que se llevaron a cabo en este proyecto, la cartografía virtual del paisaje se convirtió en un elemento clave.

\footnotetext{
${ }^{1}$ Universidad de la República, Centro Universitario Regional del Este, Departamento de Territorio, Ambiente y Paisaje, sede Maldonado, Uruguay, https://orcid.org/0000-0002-8025-9172; ${ }^{2}$ Universidad de la República, Facultad de Arquitectura, Diseño y Urbanismo, Instituto de Diseño, Programa Paisaje y Espacio Público y Centro Universitario Regional del Este, Departamento de Territorio, Ambiente y Paisaje, sede Maldonado, Uruguay, https://orcid.org/00000003-1196-8175; ${ }^{3}$ Universidad de la República, Facultad de Arquitectura, Diseño y Urbanismo, Departamento de Informática Aplicada al Diseño, Montevideo, Uruguay, https://orcid.org/0000-0002-7895-3026 y ${ }^{4}$ Universidad de la República, Centro Universitario Regional del Este, sede Rocha y Sistema Nacional de Investigadores, Área Geociencias del Programa de Desarrollo de las Ciencias Básicas, Uruguay, https://orcid.org/0000-0003-1955-7821. * Correo de contacto: analauragoni@gmail.com
} 


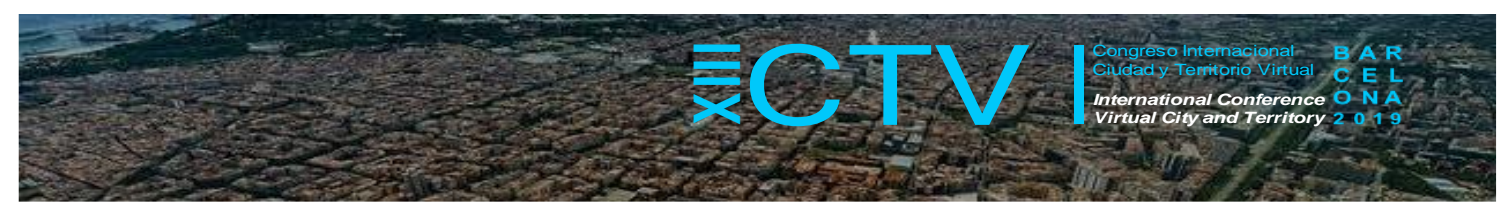

La multiplicidad de aprendizajes que se posibilitan y despliegan en este ambiente virtual está relacionada con el modo contemporáneo de conocimiento mediante el cual el mundo se interpreta como una compleja red de relaciones. El niño contemporáneo que navega a través de Google Earth posee ya una percepción visual del paisaje terrestre radicalmente distinta al niño de siglos anteriores. La idea general de paisaje como construcción cultural se ve unida a la sensación de disfrute en la apreciación de la imagen de un territorio. Vivimos en una época de cambios acelerados en la forma en que percibimos esta intrincada red de conexiones que caracteriza la era digital, por lo cual la búsqueda de nuevas formas de aprender a partir de estos territorios virtuales emerge como campo de investigación urgente y actual.

\section{Abstract}

The goal of this contribution is to expose the design and implementation of a digital platform called Pedagogic Landscapes Platform (PLAPP because of Spanish acronym), online at http://www.plapp.edu.uy, directed to children in school age and built from remote sensing and visualizing technological gadgets. This platform exposes the results of a research about local landscape perception among children from a school located in the East of Uruguay. Digital photography, drone flights and participative cartography were applied as innovative tools in order to foster landscape comprehension from different scales and approaches.

The interdisciplinary team, composed by architects, anthropologists, landscape designers and professors worked together with school teachers and children from a rural school as a pilot experience. Research developed over a 12month period. Obtained results were adapted to a communication and content format specifically tailored to school population. We developed a digital platform that exhibited several viewpoints with 360-degree vision capabilities, incorporating also sensible points that deployed additional information about the place and/ or topic. Each viewing station has a help menu in order to facilitate navigation across the available options, including orbiting from a viewpoint, displacement to another location, image zooming and capture from a contextual menu. Deployable cards containing information related to native plant trees and birds (related to the local street nomenclator) were generated by the research team and integrated into the platform, together with drawings, texts and audios generated by the children. In addition, a time lapse study was developed, based on aerial photographs provided by the local Geographical Service from 1943, 1966, 1985 and current satellite imagery, which allowed the team to develop the morphing technic by the implementation of an historical path that promote the identification of landscape transformation through time.

The unmanned aerial vehicle technology allowed the capture of high-quality video and still images (4K $3840 \times 2160$ pixels) in locations inaccessible from the ground. Aerial views covered a vast portion of the territory and allowed the generation of comprehensive panoramas, overviews, landscape markers and control points. The aerial photograph survey was conducted by circular trajectories at different fly heights and static panorama captures with varying viewing angles. Another development included the merging of such panoramas to generate a semi-spherical image which can be accessed and interactively navigated from a web browser. Such interactive image is the result of merging together 27 flat images. In addition, a version for touch sensitive devices was also developed.

The methodological approach is linked with the Learning Analytics concept by evaluating the impact of new technologies introduction to learning capabilities. It seeks to analyze data recovered from the activities proposed by the project in order to define and identify any problems or unforeseen issue that could prevent the adoption of such new learning technologies. Content and speech analysis were included in the Proyect, allowing the identification of meaningful data about the interaction between children and new technologies related language expressions. Among developed activities, virtual landscape cartography emerged as a key element.

The vast array of learning possibilities promoted by this virtual environment is strongly related with current ways of knowledge and information exchange in which the world is perceived as a complex network of interactions. A XXIth Century child who navigates through the Google Earth engine holds a visual perception of earth landscapes that is dramatically different from the perception of children from last decades and centuries. The general idea of the landscape as a cultural construction is accompanied by a pleasant sensation in viewing a territory. We are experiencing an era of abrupt and rapid changes in the way we perceive this intricate network of relationships and links that characterize the digital era. In this context, the search for new ways of teaching and learning from tools such as virtual territories emerges as a needful and promising field of research.

Palabras Clave: tecnología; paisaje; aprendizaje; plataforma digital

Key words: technology; landscape; learning; digital platform 


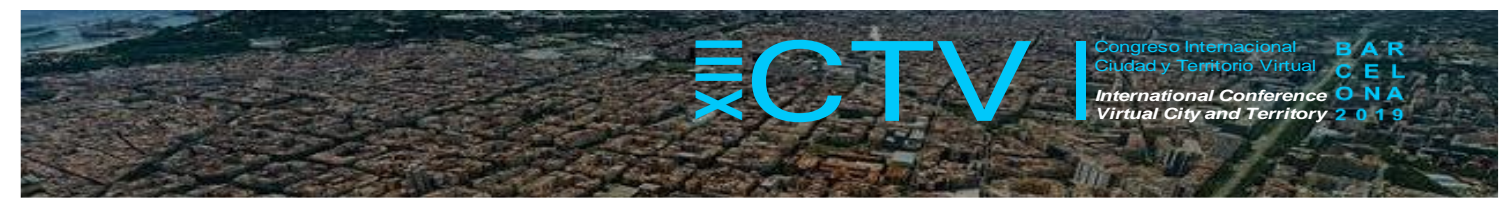

\section{Introducción}

La plataforma que se presenta en el artículo es uno de los productos finales de la investigación desarrollada en los años 2017 y 2018 que tuvo como marco procesos de enseñanzaaprendizaje en etapa escolar que consideran al paisaje como espacio estimulador del aprendizaje y vehículo para la aplicación de nuevas herramientas tecnológicas. La investigación fue financiada por la Agencia Nacional de Investigación e Innovación (ANII) y por la Fundación Conectividad Educativa de Informática Básica para el Aprendizaje en Línea (CEIBAL) en un llamado concursable del Fondo Sectorial de Educación (FSED) de Uruguay. La misma fue titulada: El paisaje como mediador de nuevas pedagogías y tecnologías y llevada adelante por el equipo de investigadores autor del artículo. Participaron educandos y educadores de la Escuela Rural №88 Faro José Ignacio, quienes indagaron en la percepción del paisaje local y fueron partícipes del diseño e instrumentación de la plataforma digital. El acercamiento al área se propuso a través de herramientas tecnológicas de sensoramiento y visualización remota para establecer distintos parámetros del paisaje, tales como vínculos entre escala y detalle, recorridos cotidianos y apreciación de ecosistemas locales. La incidencia de incorporar estos recursos fue evaluada mediante el análisis de relatos y dibujos realizados por los niños y la comparación de cartografías de paisaje previas y posteriores al uso de los dispositivos tecnológicos. Los resultados se volcaron en formato comunicacional y de contenidos adecuado a la población escolar y se sintetizaron en la plataforma digital. Por su diseño metodológico, este proceso-producto es replicable y, como tal, se pretende extender la experiencia a futuro con nuevos proyectos en otras escuelas del país.

En el ámbito internacional existen antecedentes de investigaciones y trabajos acerca del paisaje como mediador de nuevas pedagogías en la infancia. En Europa, a partir del Convenio Europeo del Paisaje del año 2000, los países se comprometieron a fomentar una formación específica desde la etapa escolar a las universidades, a tratar los valores asociados al paisaje y las cuestiones relativas a su protección, gestión y planificación (Nogué, 2011). En América del Norte, también en el cambio de milenio comienzan a surgir publicaciones académicas que revalorizan y rescatan el potencial transformador del paisaje con nuevas reflexiones disciplinares (Corner, 1999). En Latinoamérica, a partir de la Iniciativa Latinoamericana del Paisaje (LALI) firmada en el Congreso de la International Federation of Landscape Architects (IFLA) en Medellín, del año 2012, comienza a desarrollarse una concientización hacia la necesidad de la educación en paisaje como proceso bottom-up.

Asimismo, hay antecedentes de estudios asociados con la percepción ambiental y del paisaje, vinculados a la edad física de las personas y en particular de la niñez. En esta etapa, el paisaje tiene un rol fundamental en la estructuración del sentido del lugar, en la identidad y en las relaciones afectivas del niño con el espacio que habita. La educación en paisaje desde las primeras etapas de la vida reconoce los vínculos perceptivos inevitablemente establecidos con el entorno y la interdependencia con la sociedad (Busquets, 2011). Un antecedente local importante para el presente trabajo fue la investigación La ciudad inteligente. Un palimpsesto digital, llevada adelante a partir del año 2014 por el equipo del Departamento de Informática aplicada al Diseño de la Facultad de Arquitectura, Diseño y Urbanismo de la Universidad de la República. En 2014-2015, emulando la experiencia de Google Street View se llevó a cabo en el Paisaje Industrial Fray Bentos, recientemente declarado Patrimonio de la Humanidad por UNESCO, un sistema de navegación 360 grados desde el aire, disponible en http://www.patrimonioanglo.com. Este sistema permite realizar un recorrido virtual sobrevolando el sitio a modo de Skyview. Se obtiene un entorno interactivo donde el usuario puede moverse 


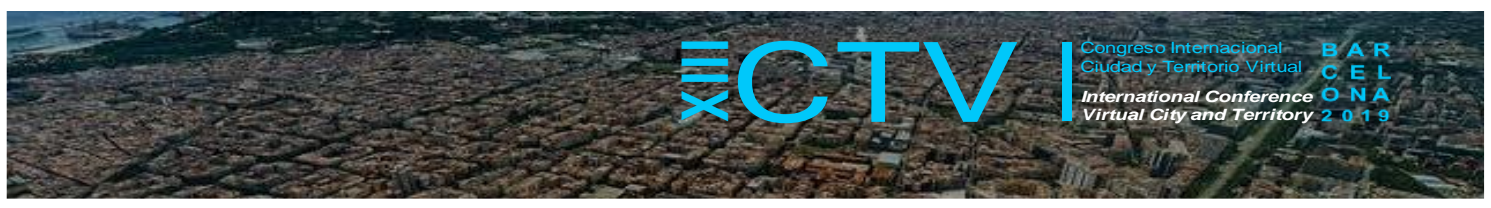

libremente y obtener información gráfica y escrita, a través de la lectura de cuadros de información asociados a edificios y lugares de interés que permite la navegación aérea. Respecto a los drones en la educación, en el ámbito local se han desarrollado experiencias anuales en el día de la Ciencia y la Tecnología en diferentes escuelas y liceos de la ciudad de Montevideo (García Amen Ed, 2017). En la actualidad, la superposición de flujos informacionales, redes de comunicación y soportes materiales e inmateriales para el flujo de conocimiento, conforma una estructura dinámica creciente, que se reescribe permanentemente a la manera de un palimpsesto digital. La ciudad inteligente puede concebirse desde una perspectiva territorial pero también desde una perspectiva socio-tecnológica contemporánea, en su permanente interacción con las tecnologías de la información y la comunicación y en su vinculación global como sistema de sistemas (García Amen y Payssé Álvarez, 2016).

\section{Diseño y contenidos de la plataforma digital}

La plataforma digital fue titulada PLAPP, surgiendo su nombre en forma colectiva durante la ejecución del proyecto, ya que cada vez que se realizaba un vuelo de dron con la presencia del colectivo escolar, los niños aplaudían al dispositivo de forma espontánea, por lo cual el sonido del aplauso -plapp-se convirtió en recurrente en las actividades del proyecto y finalmente devino en el nombre de la plataforma, disponible en http://www.plapp.edu.uy (Figura 1).

\section{Figura 1. Plataforma Paisajes Pedagógicos: plapp.edu.uy}

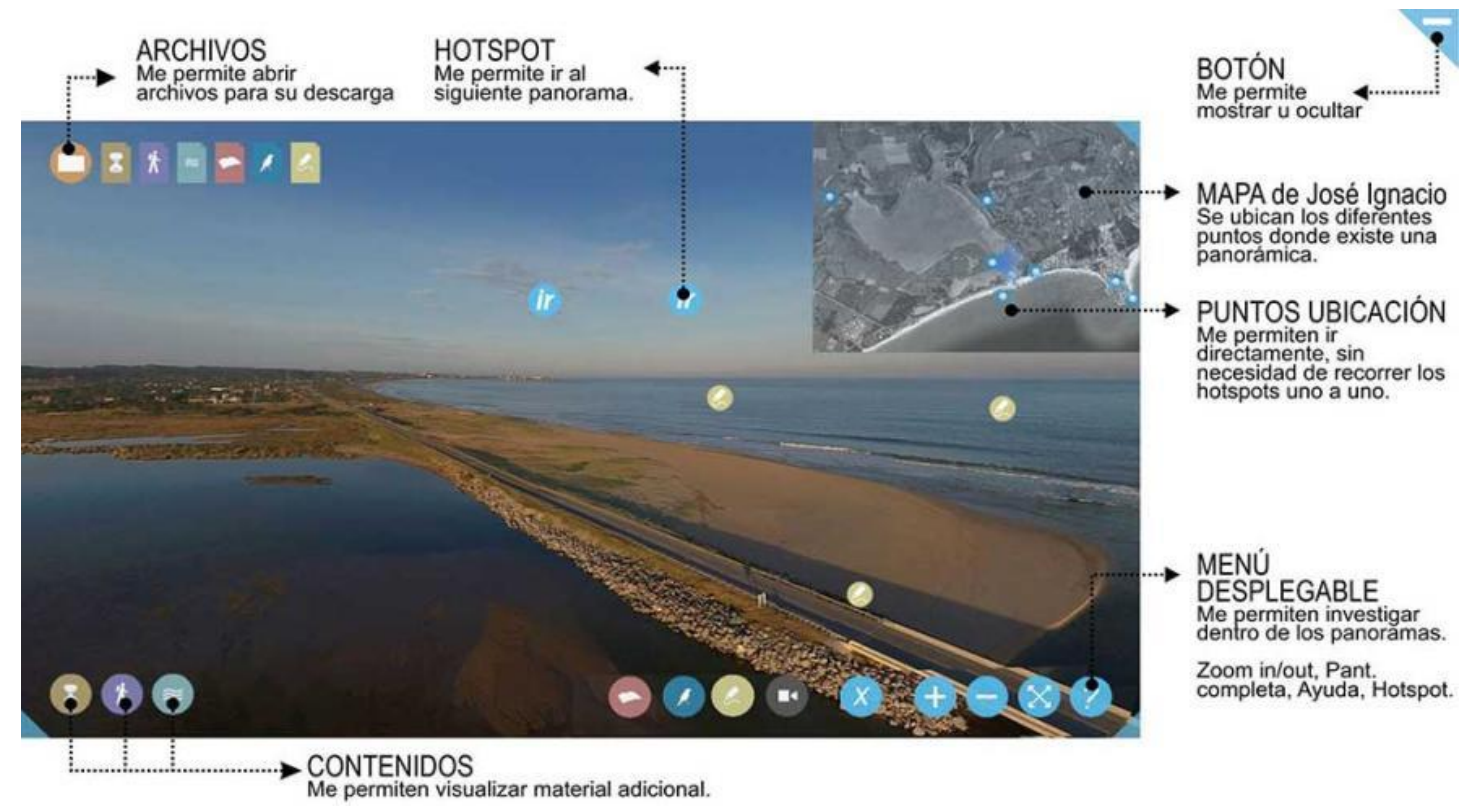

Fuente: Elaboración propia.

Asimismo, PLAPP se puede interpretar como sigla en español de PLAtaforma Paisajes Pedagógicos y al mismo tiempo en inglés como Pedagogic LAndscaPe Platform.

\subsection{Navegación 360 grados desde el aire}

Se desarrolló en la plataforma un sistema de varios puntos de observación con capacidad de navegación aérea en 360 grados y con puntos sensibles que despliegan información adicional. Las estaciones cuentan con ayudas para la navegación y posibilidades de orbitar, cambiar de 


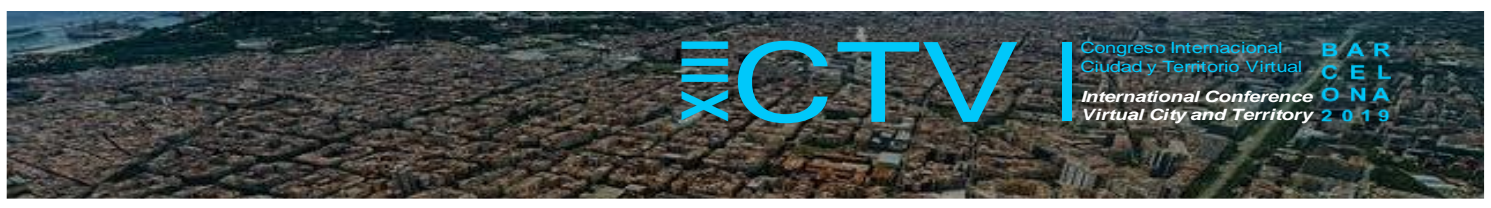

ubicación, ampliar y capturar la imagen, a través de menús auxiliares. Para construirlo se realizó un registro fotográfico a partir de vuelos circulares a distintas alturas desde un dron DJIPhantom 4 con cámara de $4 \mathrm{~K}$ y tomas panorámicas fijas con diferentes ángulos de cámara. Se generaron así panoramas de varias imágenes que luego se asociaron para formar un casquete semiesférico a los efectos de ser navegado interactivamente en la web.

La imagen esférica que se despliega en pantalla resulta de la composición de 27 imágenes planas. Al navegar en la plataforma se abre la posibilidad de salir de un punto de vista a otro (hotspot ir) transitando entre nodos, navegando sobre el territorio interactivamente desde el aire. Se configuró una versión para pantalla táctil en la que los desplazamientos son los habituales en esos dispositivos, además de la versión convencional para pantalla y mouse.

La tecnología de vehículos aéreos controlados a distancia permitió obtener video e imagen de alta calidad (4K $3840 \times 2160$ pixels), desde ubicaciones normalmente inaccesibles a nivel suelo (Figura 2). Las tomas aéreas cubrieron áreas del territorio y generaron panoramas comprehensivos, obteniendo visiones de conjunto, referencias y nodos de control.

Figura 2. Procedimientos de registro desde el aire

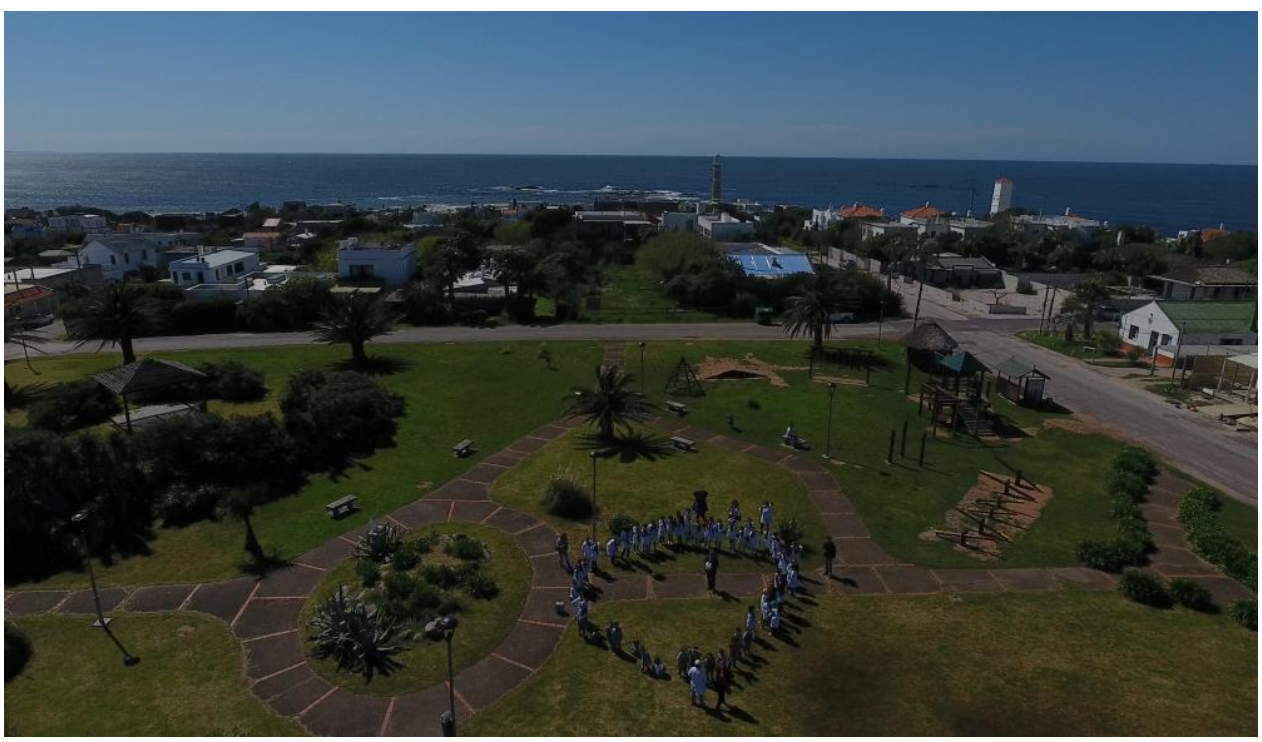

Fotografía: Marcelo Payssé Alvarez. Octubre de 2017, José Ignacio, Uruguay.

\subsection{Dibujos y relatos de escolares}

Se asociaron a la plataforma la totalidad de los dibujos realizados por los niños de la Escuela №88 durante las actividades del proyecto, bajo el ícono del lápiz se pueden encontrar sobre el Skyview (Figura 3).

Asimismo, se asociaron relatos escritos en el aula luego de las salidas de campo realizadas, con el fin de interpretar datos e identificar elementos problemáticos o desafíos en la apropiación y el manejo de los dispositivos tecnológicos. La evaluación de aprendizajes se realizó a través de la metodología de Learning Analytics, por medio del análisis de contenido y análisis de discurso para capturar datos significativos en las interacciones entre los niños, el lenguaje utilizado y las tecnologías incorporadas (Brown, 2012). 


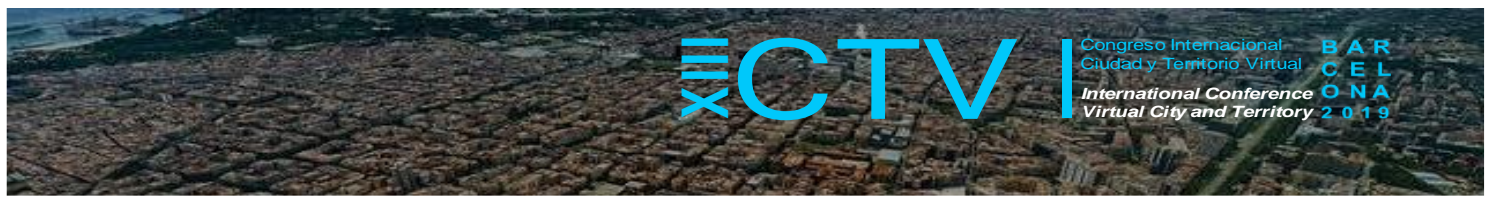

Figura 3. Fichas didácticas: dibujos y relatos de escolares

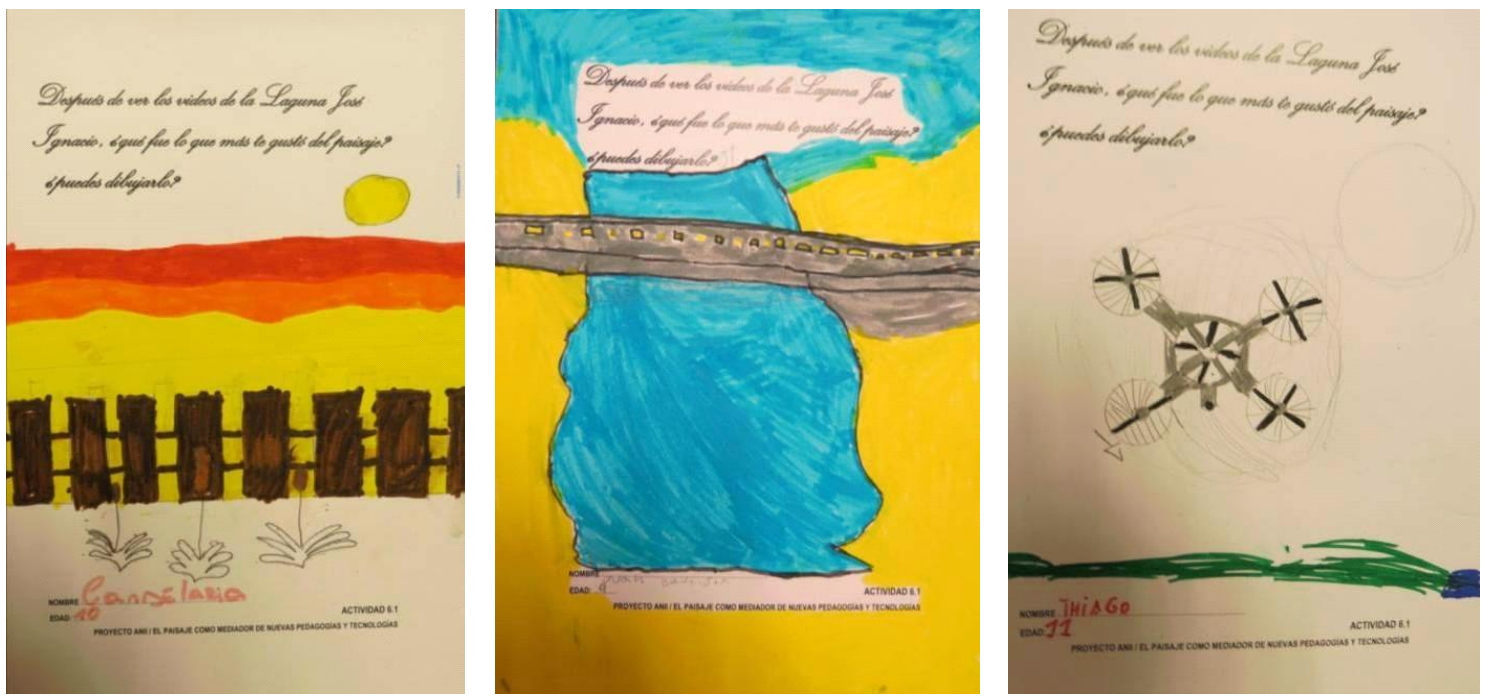

Fuente: Dibujos realizados por los niños de $4^{\circ}$ a $6^{\circ}$ año de la Escuela №88, José Ignacio, Uruguay.

En la Laguna José Ignacio se realizaron actividades de observación del paisaje, sus transformaciones en el tiempo y afectaciones de la dinámica costera provocadas por factores climáticos y el proceso de poblamiento. Se generó un registro aéreo de las conexiones entre laguna y océano, como también de los elementos construidos en esa zona litoral que impactan las dinámicas del paisaje. Posteriormente se realizaron en aula dibujos de la morfología de la laguna que se sintetizaron en un mosaico de siluetas (Figura 4).

La producción de los niños fue analizada cuantitativa y cualitativamente con la intención de identificar elementos que podrían descubrirse en el proceso de aprendizaje, como también las herramientas y los conocimientos que se incorporaron. El análisis de datos y discusión aparece publicado en un artículo recientemente publicado, elaborado por el equipo de investigadores (Goñi, A. L. et al., 2019).

Figura 4. Mosaico de siluetas de la Laguna José Ignacio.

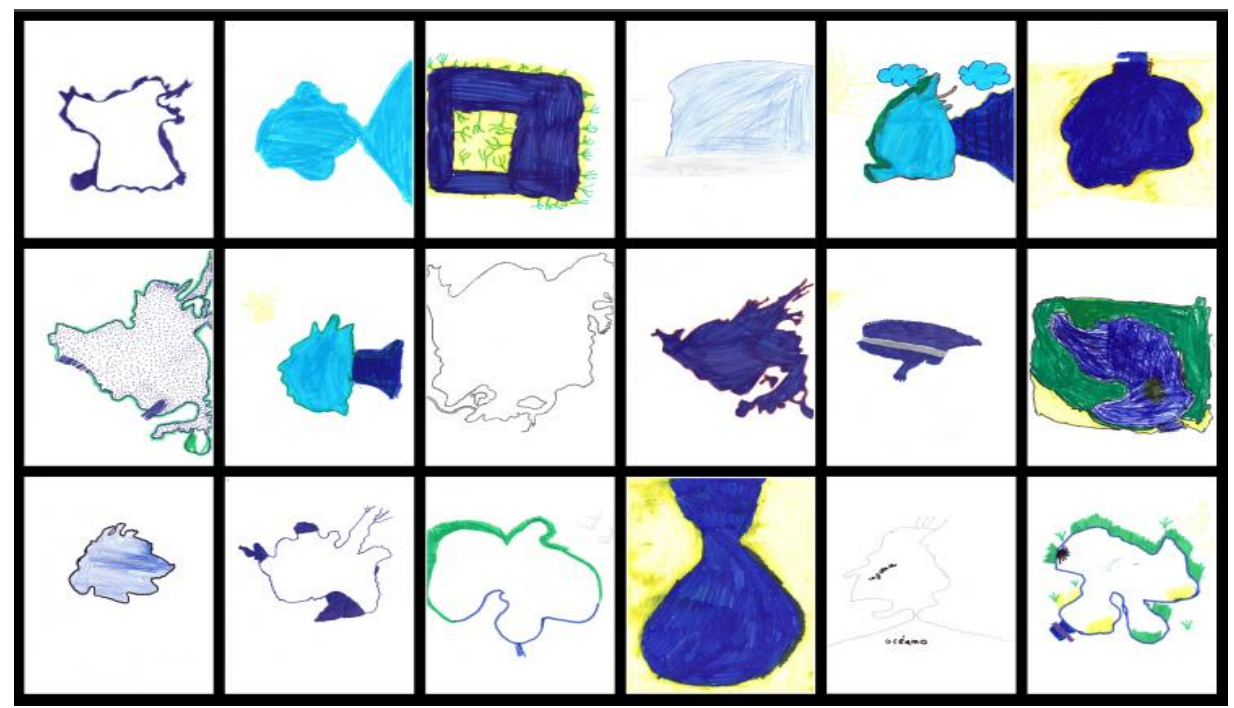

Fuente: Dibujos realizados por los niños de 4ํ a 6ำ año de la Escuela №88, José Ignacio, Uruguay. 


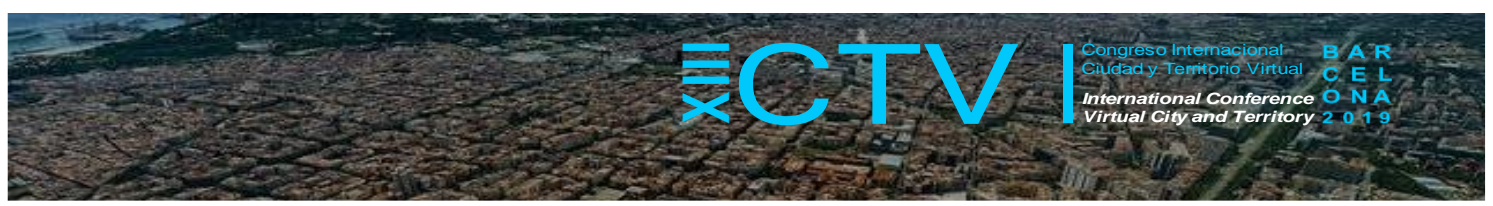

\subsection{Morphing histórico aéreo}

Se trabajó con fotografías aéreas históricas del cuerpo lacustre, de los años 1943, 1966, 1985 y fotografías satelitales más actuales, a los efectos de reconocer las transformaciones del paisaje en el tiempo (Figura 5). Se aplicó la técnica del Morphing para realizar un recorrido virtual por la historia del lugar, permitiendo ver en las fotografías más antiguas, correspondientes al vuelo Trimetrogon del Servicio Geográfico Militar de Uruguay de 1943, las dunas de arena como matriz dominante del paisaje, que luego se van transformando por medio de la forestación y antropización progresiva del territorio, modificando en su totalidad aquel paisaje histórico hasta llegar a las fotografías satelitales actuales, con el ícono del reloj de arena. Esta técnica sirvió para visualizar la transformación territorial de una forma ágil y continua, para facilitar la comprensión por parte de los niños del proceso histórico de cambios en el paisaje que van más allá de su lapso de vida, permitiendo a los maestros trabajar temas relativos a la historia del lugar y al impacto de la antropización en el sitio.

En las actividades vinculadas al trabajo concreto con estas fotografías aéreas en el aula, surgieron diálogos entre educandos y educadores que explicitaban otros diálogos de los escolares con sus padres y abuelos en que relataban cuentos y anécdotas de los anteriores habitantes del sitio pertenecientes a sus propias familias, a partir de la visualización conjunta en sus hogares de la plataforma, despertando así a través de esta técnica digital del morphing, a modo de viaje virtual en el tiempo, la curiosidad por conocer datos históricos en los educandos y un acercamiento lúdico a la historia, asignatura que los maestros participantes señalaron de difícil motivación. La herramienta se convierte entonces, en un medio tecnológico que despierta las ganas de aprender acerca de la historia de los paisajes locales.

Figura 5. Transformaciones del paisaje en el tiempo
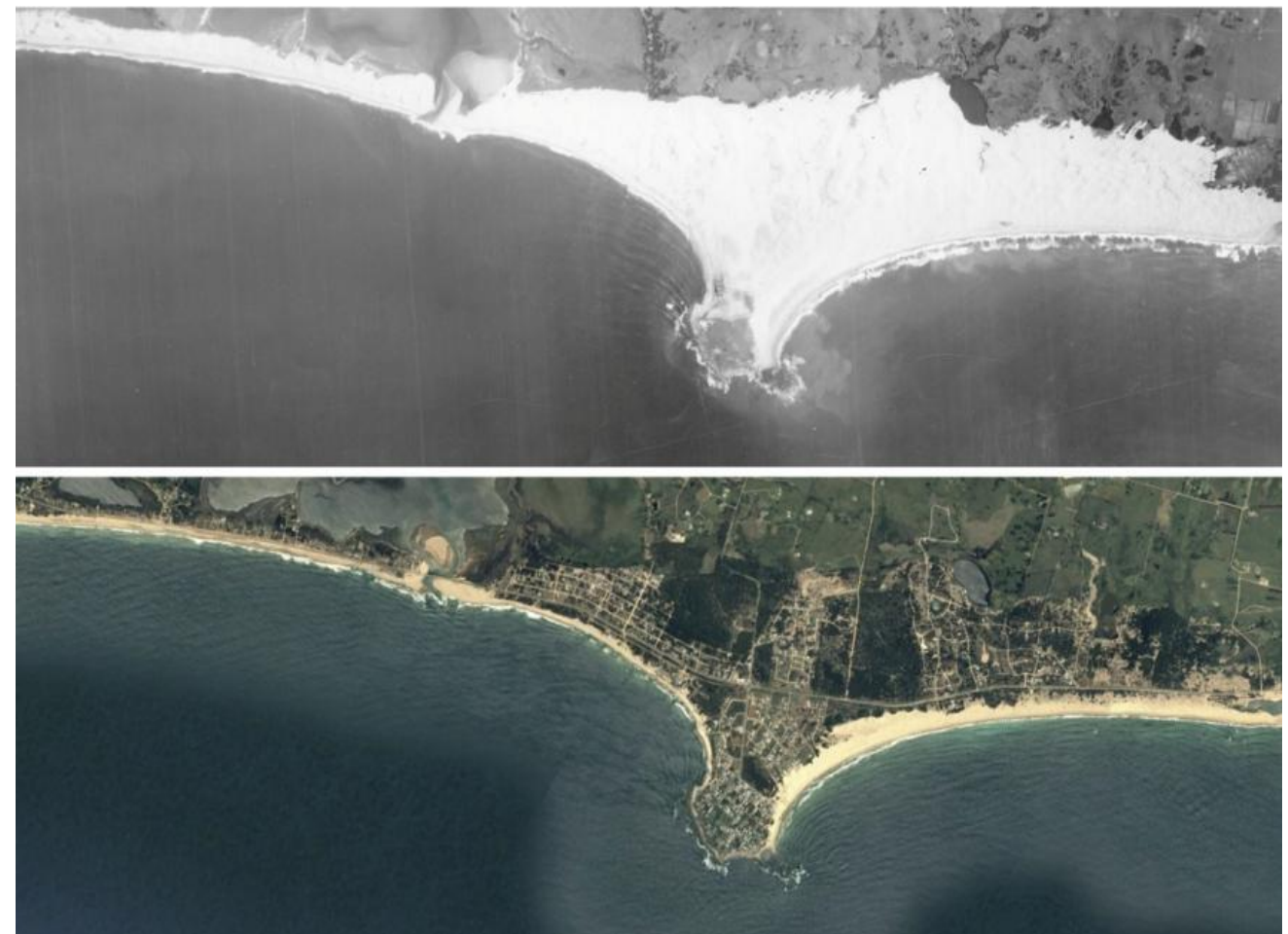

Fuente: Elaboración propia en base a Fotografía aérea SGM vuelo histórico Trimetrogon escala 1:40.000, año 1943 y Fotografía satelital de fuente Google Earth, año 2016. 


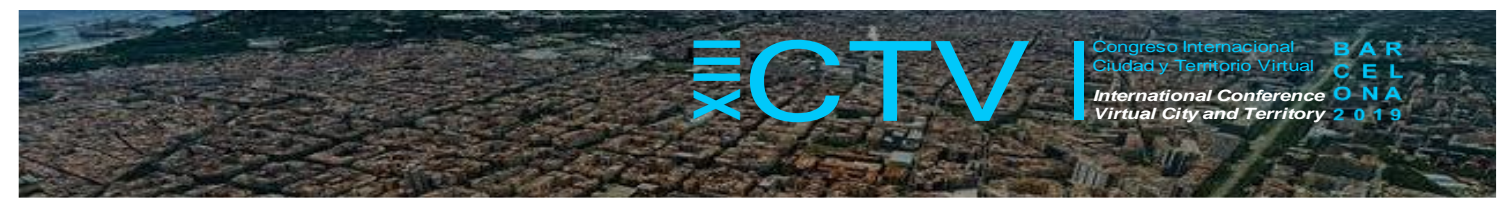

\subsection{Cartografías colectivas y audios}

Con el apoyo de fotografías satelitales actuales obtenidas de Google Earth se realizaron cartografías colectivas, identificando los componentes paisajísticos más frecuentes observados por los niños en sus recorridos cotidianos (Figura 6). Las cartografías de paisaje son un desafío a construir, las representaciones cartográficas se convierten en un medio para aprender a aprender, un reto en sí mismo a explorar (Sala, 2013). Se vuelve a utilizar la técnica del morphing para ir navegando en forma virtual entre una y otra cartografía. Al analizar los dibujos creados aparece el código gráfico, distinto al de la lectoescritura. Dibujando el entorno vital se ponen de manifiesto los puntos de referencia espaciales de los niños, los diversos grados de conocimiento del territorio donde habitan y sus valoraciones hacia el paisaje que perciben. En el análisis comparativo de actividades realizadas antes y después de la interacción con los videos grabados desde el dron, se evidencian cambios en los elementos dibujados más frecuentemente. El cambio de horizonte normal a horizonte aéreo es registrado en los dibujos como una ampliación de la capacidad de ver nuevos componentes del lugar.

Figura 6. Cartografías colectivas

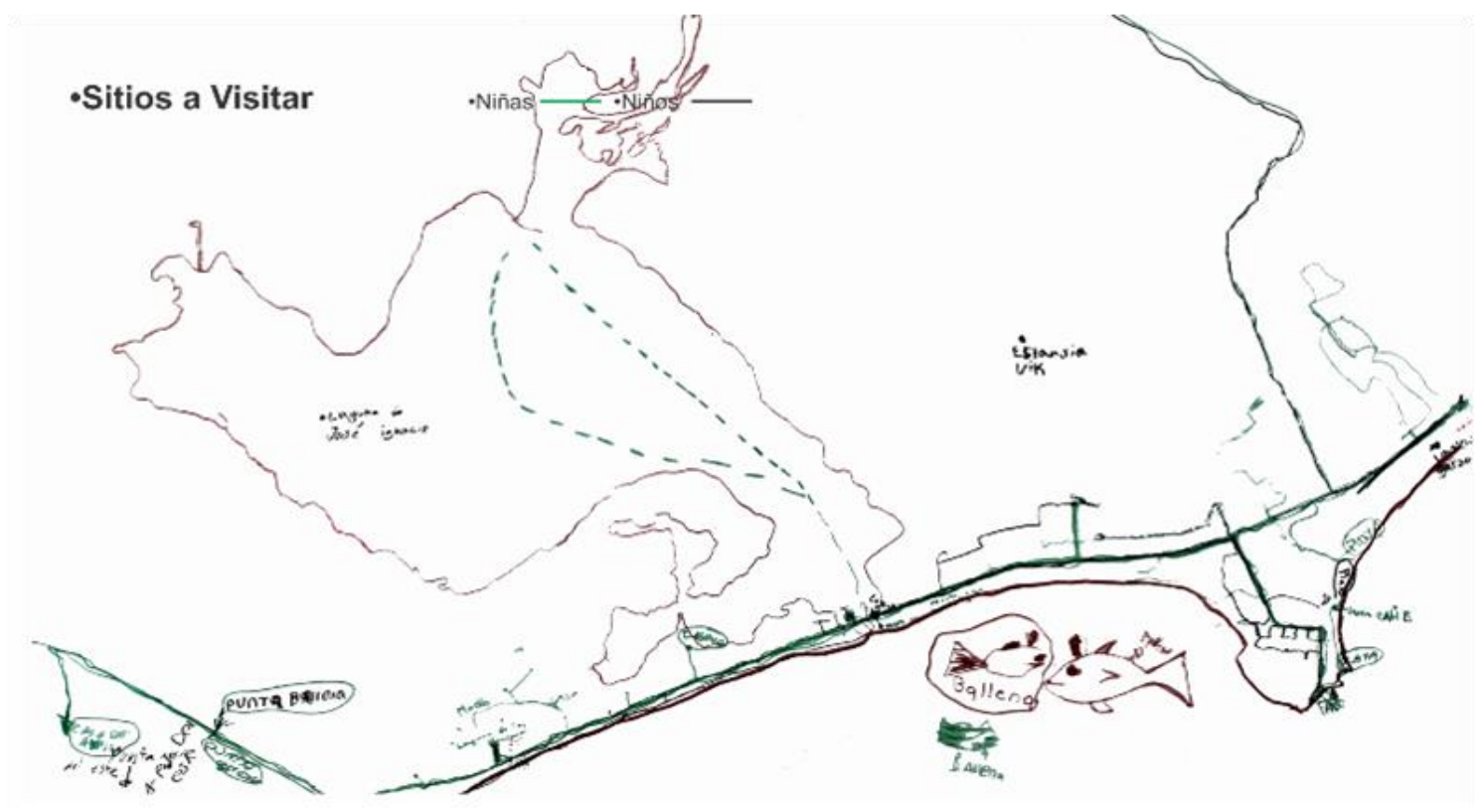

Fuente: Cartografía elaborada por niños de la Escuela №88 en base a fotografía satelital de Google Earth de la región de la Laguna José Ignacio.

El cuento de Italo Calvino de su libro Las ciudades invisibles, titulado Esmeraldina, habla de una ciudad imaginaria donde habitan golondrinas, siendo éste el nombre de la calle de la Escuela $\mathrm{N}$ ㅇ8 (Calvino, 1994). El audio con el cuento leído por una niña de una Escuela Pública de Montevideo, Uruguay, es subido a la plataforma. De esta forma se generan vínculos entre niños de diversas ciudades del país promovidos por el espacio virtual compartido.

\subsection{Fichas de aves y fichas de vegetación nativa}

En la actividad que se dedica al Nomenclátor de la localidad de José Ignacio se trabaja acerca de las aves de la región. Durante esta actividad se realiza un recorrido virtual, sobre mapas, por cada una de las calles de José Ignacio y se identifica el ave, se dibuja y se lee información 


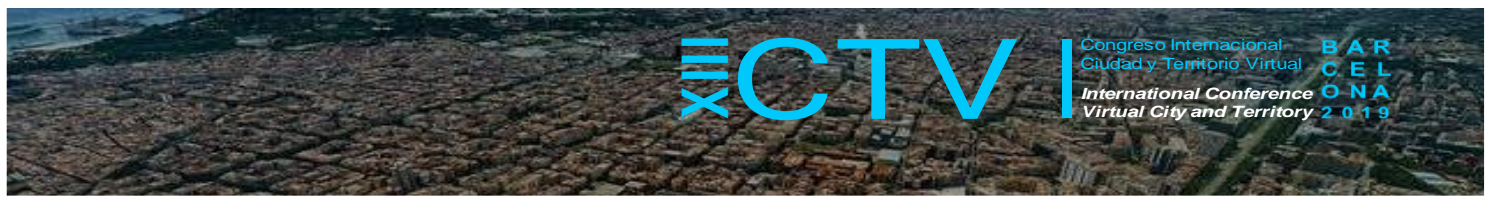

del libro: Guía para la identificación de las aves de Argentina y Uruguay (Narosky \& Yzurieta, 2003). La información acerca de las características de cada ave, su comportamiento y su hábitat, es asimilada por los niños y reproducida en sus dibujos. Se realizan por parte del equipo de investigadores una serie de Fichas de Aves, con datos de las aves que figuran en el Nomenclátor del pueblo, que se suben a la plataforma y se permiten bajar en formato pdf para transformarse en fichas didácticas coleccionables, bajo el ícono del ave (Figura 7).

Figura 7. Ejemplo de Ficha de Aves. Nomenclátor José Ignacio

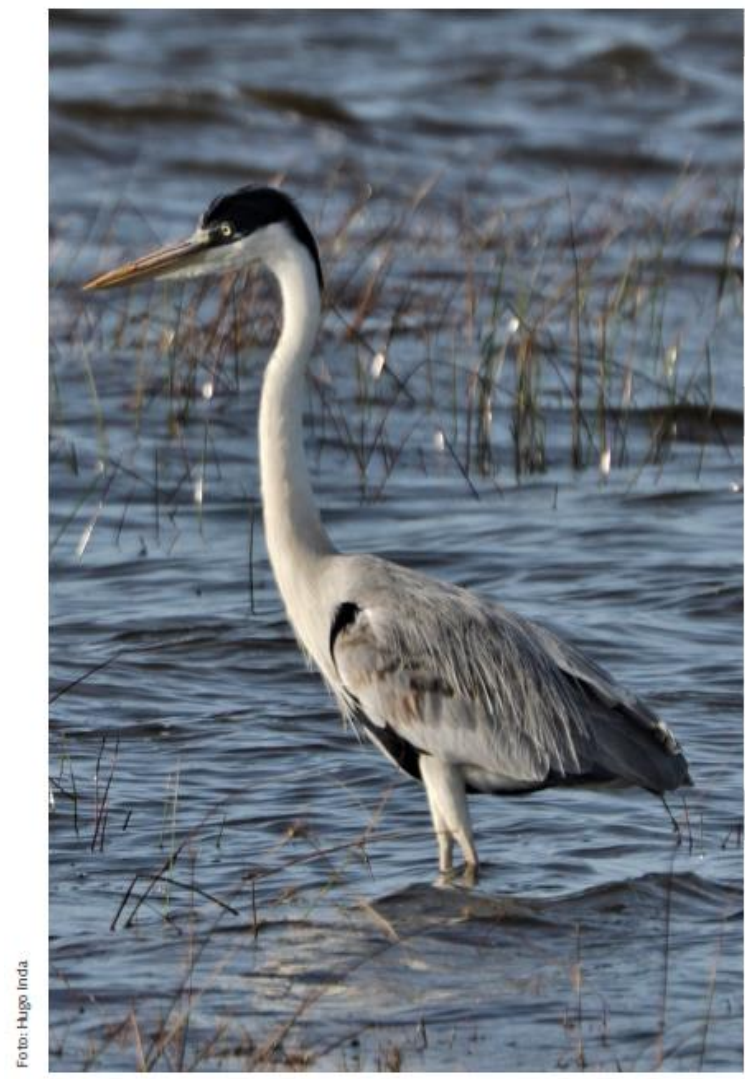

\section{GARZA MORA \\ Familia: Ardeidae \\ ¿COMOES?}

Es la garza de mayor tamaño, mide unos $75 \mathrm{~cm}$. Tiene en la parte superior de la cabeza plumas alargadas y negras. Su dorso es gris y el resto del cuerpo de color blanco.

\section{¿COMO SE COMPORTA?}

Se la observa sola 0 en pareja. Se suele posar sobre los árboles 0 en la orilla del río para acechar a sus presas. Su vuelo es pausado y majestuoso. Como alarma emite una voz áspera y grave.

Se alimenta principalmente de peces y de culebras, ranas, insectos, anfibios y crustáceos.

El nido tiene la forma de una plataforma grande, realizada con juncos y agregado de palitos o ramitas.

\section{¿DÓNDE LO ENCONTRAMOS?}

Habita en ambientes acuáticos: esteros, lagunas, charcos, islas, orillas de arroyos y de rios

Lo encontramos desde Panamá hacia el cono sur.
Ardea cocoi

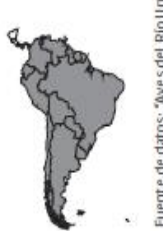

Fuente: Elaboración propia en base a la Guía para la identificación de las aves de Argentina y Uruguay (Narosky y Yzurieta, 2003) y fotografías de Hugo Inda.

A partir de una salida didáctica a la sierra de Carapé y de la actividad de reconocimiento de flora indígena realizada en campo, se ensaya en el dibujo de especies vegetales con los niños. Se trabaja y reflexiona a partir de la vegetación observada y de los componentes de la misma que destacan en el paisaje (flores, frutos, cortezas, ramillas, etc.) y se muestran en el aula los dibujos en acuarela de las diferentes especies realizados por Pedro Cracco y reproducidos en el libro Flora Indígena del Uruguay (Muñoz et al., 1993).

Se realizan luego, por parte del equipo de investigación y con el asesoramiento del Ing. Agr. Pablo Ross, una serie de Fichas de Vegetación Nativa que aportan información de cada especie observada en la salida didáctica y de su comportamiento y su hábitat, las cuales se adaptan en formato comunicacional dirigido a los niños y se incorporan a la plataforma digital (Figura 8). 


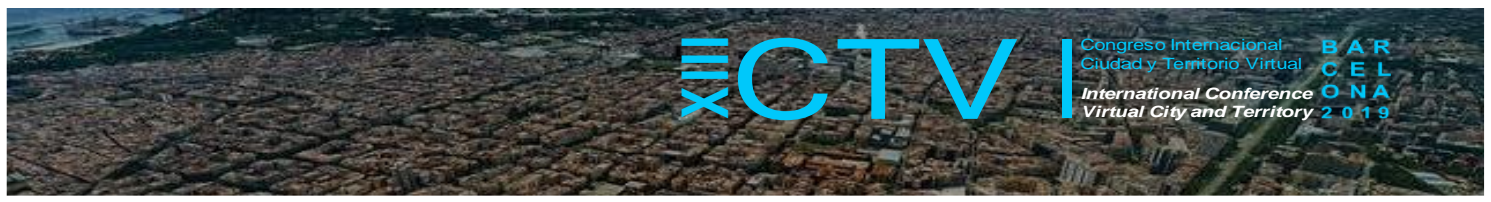

Figura 8. Ejemplo de Ficha de Vegetación Nativa

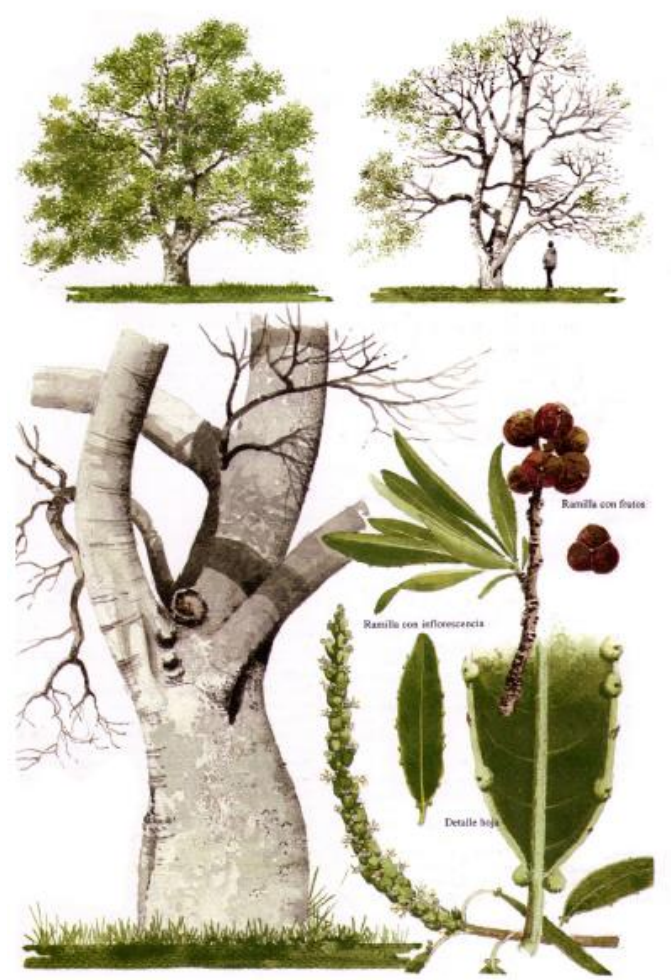

CURUPI O ARBOL DE LA LECHE Sapium Montevidense

Familia: Euphorbiaceae

¿COMOES?

Es un árbol robusto de entre 7 y $8 \mathrm{~m}$ de altura con tronco algo tortuoso de color castaño, corteza fina, persistente y copa redondeada. Su follaje es semipersistente a caduco de color verde claro. Sus hojas son simples, alternas, con forma de elipse y de $12 \mathrm{~cm}$ de largo con borde finamente aserrado. Las hojas tienen dos pequeñas glandulas productoras de látex. Su fruto es tricoco de $1 \mathrm{~cm}$ de diámetro, rojizo cuando está maduro.

\section{¿COMO SE COMPORTA?}

Florece en primavera con flores pequeñas sin pétalos dispuestas en espigas. Tiene flores femeninas en la mitad inferior y masculinas en la parte superior.

\section{¿DÓNDE LO ENCONTRAMOS?}

Es indigena de las zonas tropicales y subtropicales. En Uruguay la encontramos en lugares bajos y húmedos.

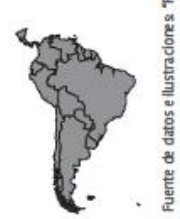

Fuente: Elaboración del equipo de investigación con el asesoramiento del Ing. Agr. Pablo Ross.

\subsection{Diseño de secciones, pruebas y ajustes}

Se realizaron pruebas del producto específico de la plataforma con los niños de la Escuela №88 a los efectos de realizarle ajustes. Se incluyó una divulgación regional al invitar a otras escuelas rurales a participar de las actividades finales. Los investigadores recibieron comentarios de los niños acerca de observaciones efectuadas sobre la plataforma digital y sus potencialidades desde la mirada del usuario. Asimismo, se recibió una evaluación escrita por parte de las maestras, que incluyó apreciaciones de los efectos reconocibles en el aprendizaje de las actividades realizadas en el proyecto: trabajo en equipo, desarrollo de la oralidad, surgimiento de capacidades de exposición y debate en los niños, trasmisión de lo que aprendieron a sus educadores y generación de un conocimiento fundamentado. En relación a la interface de uso, se evaluó como entendible y de buena diagramación, sin encontrar dificultades mayores en el uso cotidiano. En relación a los íconos y el lenguaje utilizado, se evaluaron de fácil comprensión y rápida asimilación por los niños. La apertura pública de la plataforma se ponderó positivamente, en contraste con ámbitos más cerrados y específicos de la comunidad educativa. En cuanto a los elementos que resultaron más didácticos para actividades con los niños se resaltaron en primer lugar las filmaciones 360 grados, en segundo lugar, la presencia de los dibujos y relatos de todos los niños participantes en la plataforma, en tercer lugar, las fichas de aves y de vegetales diseñadas en formato apropiado al uso escolar, en cuarto lugar, los mapas colectivos sobre fotografías satelitales y por último el morphing de fotografías históricas aéreas de la laguna. Asimismo, se incluyeron comentarios sobre la diversidad de componentes de la plataforma como elemento a resaltar, diversidad como 


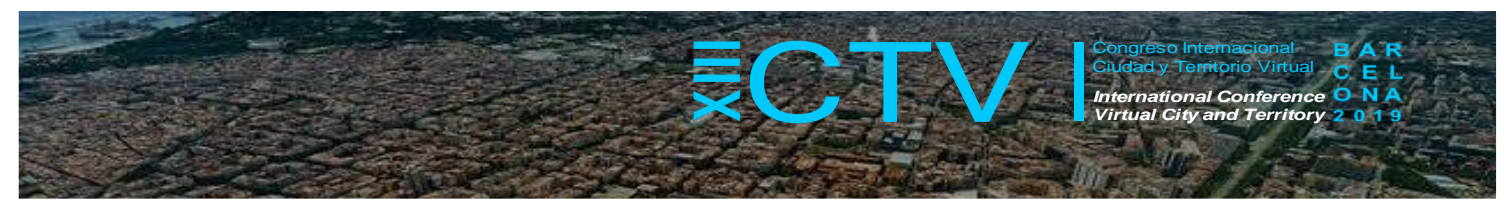

generadora de mayor curiosidad en los usuarios durante su navegación cotidiana, al ser productos variados (imagen fija, sonido, video, etc), combinados en una plataforma única, dirigida no solamente a los educandos sino a los educadores a través de las secciones específicas anteriormente detalladas. Se destacó en la evaluación realizada por las maestras, que cada uno de los elementos incluidos en la plataforma tiene un aporte específico y enriquece la labor de niños y docentes.

Asimismo, en el diseño de la plataforma se incluyen distintas secciones conteniendo la definición del proyecto, alcances, créditos, presentación del equipo de investigación y colaboradores y los avances periódicos de las aplicaciones desarrolladas. Por tratarse de un espacio dinámico se van adosando con el tiempo nuevas secciones, como ser la recientemente creada bajo la denominación Artículos publicados, donde se encuentran publicados los resultados completos de la investigación inicial en el artículo titulado El paisaje como mediador de nuevas pedagogías y tecnologías, publicado en junio de 2019 en la revista indexada digital de la Universidad de Boyacá, Colombia, Designia 6(2), 25-53. A la brevedad se publicará bajo una nueva pestaña a denominar Congresos, el texto de la presente ponencia en el XIII Congreso Internacional de Ciudad y Territorio Virtual de Barcelona 2019.

\section{Confluencias finales}

El primer resultado a señalar es la verificación del potencial del paisaje como instrumento mediador en el desarrollo de nuevas pedagogías y en la incorporación de nuevas tecnologías.

En segundo lugar y en relación al proceso desarrollado, se trabajó en la promoción de procesos de aprendizaje activo ubicando a los niños como principales protagonistas, colaborando en el desarrollo de competencias para la acción, aportando capacidades para imaginar y motivando una disposición para la búsqueda y construcción de conocimiento. Se promovió un ambiente de aprendizaje donde se conectó lo ya conocido con lo nuevo, ayudando a establecer relaciones y conexiones.

En tercer lugar, se verificó que el uso de herramientas tecnológicas de sensoramientovisualización remotas facilita la visualización y reconocimiento de diferentes paisajes. Los nuevos dispositivos tecnológicos de sensoramiento remoto y las plataformas interactivas fueron puestos al servicio de las inquietudes de los niños, como herramientas de aprendizaje de las principales características del entorno ambiental, cultural y paisajístico local. Asimismo, se abordó el instrumento tecnológico como oportunidad de difundir los alcances de las TICs en el ámbito escolar, mediante explicaciones, experimentación y reflexiones sobre los nuevos medios aplicados al registro del paisaje.

El proyecto realizado generó acontecimientos con características particulares -múltiples, rápidos, efímeros- propios de la contemporaneidad, de acuerdo con la definición de los seis temas claves del actual milenio expuesta por Italo Calvino. La multiplicidad está vinculada con el actual modo de conocimiento por el cual el mundo se interpreta como una compleja trama de relaciones sobrepuestas, una intrincada red de conexiones (Calvino, 1999). Dicha trama implica movimientos externos e internos, una relocalización de los roles del docente y el alumno para pensar desde otro lugar y generar nuevos vínculos en el espacio escolar cotidiano. 


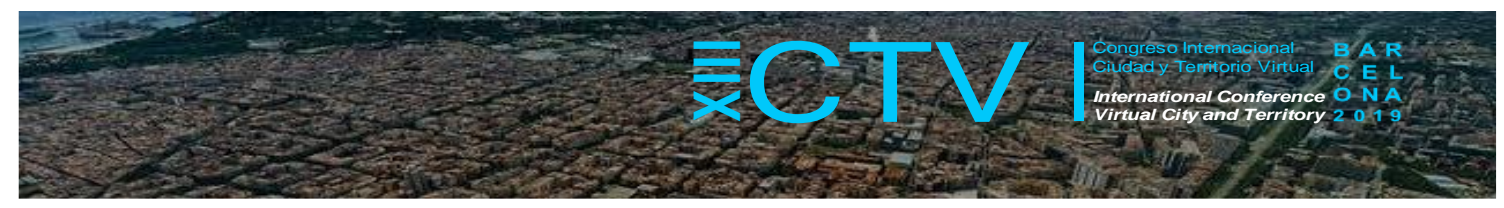

El equipo de investigación evalúa que estas herramientas tecnológicas de sensoramientovisualización remotas permiten aprendizajes significativos. Esto se verifica en esta experiencia mediante el análisis y la comparación de cartografías de paisaje y relatos escritos previos y posteriores a su utilización. La metodología aplicada busca apoyar espacios de enseñanza y aprendizaje escolar más apropiados al uso de nuevas tecnologías. En este sentido y a modo de síntesis señalamos como aportes los siguientes:

- El ensayo en el dispositivo tecnológico como estímulo al proceso cognitivo del niño que puede incidir en el aprendizaje.

- La profundización en el conocimiento del espacio territorial regional, área de hábitat de la comunidad escolar.

- La plataforma digital donde los avances se presentan en un formato comunicacional y de contenidos adecuado a la población escolar participante de la investigación.

- Se contribuyó a la incorporación de nuevas tecnologías como herramientas para la generación de conocimiento.

En las actividades que se llevaron a cabo en este proyecto, la cartografía virtual del paisaje se convirtió en un elemento clave. La multiplicidad de aprendizajes que se posibilitan y despliegan en este ambiente virtual está relacionada con el modo contemporáneo de conocimiento mediante el cual el mundo se interpreta como una compleja red de relaciones. El niño contemporáneo que navega a través de Google Earth posee ya una percepción visual del paisaje terrestre radicalmente distinta al niño de siglos anteriores. La idea general de paisaje como construcción cultural se ve unida a la sensación de disfrute en la apreciación de la imagen de un territorio (Goñi, 2012). Vivimos en una época de cambios acelerados en la forma en que percibimos esta intrincada red de conexiones que caracteriza la era digital, por lo cual la búsqueda de nuevas formas de aprender a partir de estos territorios virtuales emerge como campo de investigación urgente y actual.

La plataforma generada a partir del trabajo en la Escuela №88 se visualiza como el inicio de un recurso semejante, aunque mucho más abarcativo desde el cual los centros educativos puedan compartir el conocimiento sobre sus paisajes locales. Por lo tanto, el itinerario no ha de ser único pues si bien se encontrará orientación en objetivos similares, los trayectos serán trazados por cada caso particular. Esta experiencia contribuye a la formación de los niños y a través de ellos de sus familias. Se favorece así una mejor comprensión del hábitat y se perfila una estrategia de sostenibilidad apoyada en la comunidad escolar.

Agradecimientos: A la Agencia Nacional de Investigación e Innovación (ANII) por haber financiado el presente proyecto, FSED-2-2016-1-130781, en el marco de la Convocatoria 2016 del Fondo Sectorial de Educación (FSED). A la Comisión Sectorial de Investigación Científica (CSIC-UdelaR) por haber financiado el Proyecto "La Ciudad Inteligente; un palimpsesto digital", en el ámbito del cual se han realizado las aproximaciones tecnológicas del presente trabajo. A los consultores y colaboradores del equipo del proyecto "El paisaje como mediador de nuevas pedagogías y tecnologías": Pablo Ross, Victoria Sánchez, William Pérez, Victoria López, Fernando García, Luis Flores y Lucía Meirelles. A la directora de la escuela rural 88 "Faro José Ignacio", Maestra Silvia Rivas, como también a Nilvia Ferrizzo, maestra de $4^{\circ}$ a $6^{\circ}$ grado de la institución educativa, y a todos los niños participantes. 


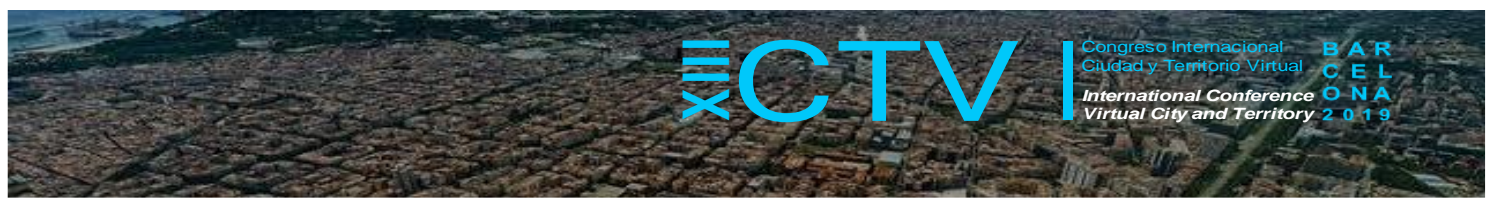

Contribuciones de los autores: Los autores han desarrollado el texto de la ponencia en conjunto.

Conflicto de Intereses: Los autores declaran que no hay conflicto de intereses.

\section{Bibliografía}

Brown, M. (2012). Learning Analytics: Moving from Concept to Practice. En Educause Review, Learning Initiative Briefing. Recuperado de http://www.library.educause.edu/resources/2012/7/ learning-analytics-moving-from-concept-to-practice

Busquets, J. (2011). La importancia de la educación en paisaje. En J. Nogué (Ed.). Paisatge $i$ educación (pp.378-380). Olot, España: Observatorio del Paisaje de Cataluña.

Calvino, I. (1999). Seis propuestas para el próximo milenio. Madrid, España: Siruela.

Calvino, I. (1994). Las ciudades invisibles. Madrid, España: Siruela.

Corner, J. (Ed.). (1999). Recovering Landscape. Essays in Contemporary Landscape Architecture. New York: Princeton Architectural Press.

García Amen, F. y Payssé Àlvarez, M. (2016). La ciudad inteligente -Un palimpsesto digital-. ARQUISUR Revista, 6(10), 18-25.

García Amen, F. Ed. (2017). FOLDERS/01. Repositorio de trabajos realizados por el Departamento de Informática Aplicada al Diseño 2014-2015. Montevideo, Uruguay: Ed. Universitarias, Universidad de la República.

Goñi, A. L., Piazza, N., Payssé, M y Inda, H. (2019) El paisaje como mediador de nuevas pedagogías y tecnologías. En Revista Designia 6(2), 25-53.

Goñi, A. L. (2012). La condición del paisaje como construcción perceptiva. En M. Sánchez \& M. Fernández (Coord.) Paisaje y Entorno: reflexiones multidisciplinares (pp.68-76). Murcia, España: Biovisual.

Muñoz, J., Cracco, P. y Ross, P. (1993). Flora indígena del Uruguay: árboles y arbustos ornamentales. Montevideo, Uruguay: Hemisferio Sur.

Narosky, T. \& Yzurieta, D. (2003). Guía para la identificación de las aves de Argentina y Uruguay. Buenos Aires, Argentina: Vazquez Mazzini.

Nogué, J. (Ed.). (2011). Paisatge i educación. Olot, España: Observatorio del Paisaje de Cataluña.

Sala, P. (2013). Cartografiar els paisatges d avui i els que vénen. En J. Nogué (Ed.). Reptes en la cartografia del piasatge (pp.14-41). Olot, España: Observatorio del Paisaje de Cataluña. 